\title{
The effects of trazodone on human cognition: a systematic review
}

\author{
Ana Mafalda Gonçalves Gonçalo ${ }^{1}$ (1) · Maria Augusta Vieira-Coelho ${ }^{1,2}$ (D)
}

Received: 28 January 2021 / Accepted: 13 May 2021 / Published online: 7 June 2021

(c) The Author(s), under exclusive licence to Springer-Verlag GmbH Germany, part of Springer Nature 2021

\begin{abstract}
Trazodone is a widely used antidepressant that is also useful in the control of agitation and insomnia in Alzheimer's disease. This drug is now recognized as having a new mechanism of action, an effect on the unfolded protein response (UPR) pathway, restoring protein translation and slowing neurodegenerative progression in mice. This mechanism may have a role in dementia-modifying treatment. To explore the effects of trazodone on human cognition and to search for clinical evidence of its putative benefits in human neurodegenerative diseases, a systematic review was conducted for studies that evaluated the effect of a minimum dose of $25 \mathrm{mg}$ of trazodone daily, for at least 1 week, on cognition in adult humans. The search was run in MEDLINE, Web of Science, and CENTRAL from the Cochrane databases, yielding a total of 16 studies after selection. Overall, seven studies showed no effect of trazodone on cognition, five showed a beneficial effect by improving or reducing cognitive decline, and four evidenced impaired cognitive function. Our analysis highlights the possibility of a dose-independent dual effect of trazodone on human cognition, with acute utilization associated with impaired cognitive function and long-term use with preventing cognitive deterioration. There was no clinical evidence that trazodone could be used as a specific treatment of neurodegenerative diseases. Future studies should explore the role of trazodone in the UPR pathway and the implications in neurodegenerative diseases in humans.
\end{abstract}

Keywords All cognitive disorders/dementia $\cdot$ Alzheimer's disease $\cdot$ Memory $\cdot$ Executive function $\cdot$ Trazodone

\section{Introduction}

Although it is FDA-approved only for use in the treatment of major depression, trazodone, a drug created in the 1960s [1], is widely used off-label to control agitation and insomnia in Alzheimer's disease (AD) [2] and in anxiety, schizophrenia, bulimia, substance abuse, fibromyalgia [3], and post-traumatic stress disorder [1,4]. Additionally, it reduces behavioural and psychological symptoms in $\mathrm{AD}$ and frontotemporal dementia [2].

Trazodone is now recognized as having a novel mechanism of action, its effect in the unfolded protein response (UPR) pathway. Trazodone acts downstream of eIF2 $\alpha-P$, preventing it from reducing levels of the ternary

Ana Mafalda Gonçalves Gonçalo

mafaldagoncalo@gmail.com

1 Department of Biomedicine, Pharmacology and Therapeutics Unit, Faculty of Medicine, University of Porto, Porto, Portugal

2 Department of Psychiatry and Mental Health, University Hospital Center of São João, Porto, Portugal complex and allowing protein translation to occur [2] (see Supplementary Fig. 1). Hence, trazodone has been shown to prevent the effects of UPR overactivation observed in neurodegenerative diseases [2] and restore neuronal protein synthesis, preventing neurodegeneration in mouse models [5]. As a result, this drug appears to have utility as a novel disease-modifying treatment for neurodegenerative diseases in humans [2].

However, if trazodone has a role in preventing cognitive decline, it is important to consider whether that effect is mediated through its action in the UPR pathway, by increasing the synaptic concentration of 5-hydroxytryptamine [6], or as a result of the sleep improvement this drug offers. Establishing the effect of trazodone on cognition and neurodegeneration would have significant implications for clinical practice, given the increasing prevalence of neurodegenerative diseases and the extensive use of trazodone in this patient population.

Thus, we aimed to establish the effects of trazodone on human cognition and to determine whether there was evidence that it could be used in the treatment of neurodegenerative diseases in humans. 


\section{Methods}

Following PRISMA [Preferred Reporting Items for Systematic Reviews and Meta-Analyses] guidelines, we conducted a systematic review, without a meta-analysis, of studies that evaluated the effect of trazodone on human cognition.

Our target population was healthy or diseased adults aged 18 years or older. The diseased patients could have all types of illness, from atherosclerotic disease to psychiatric conditions. We excluded animal studies, since our focus was on the consequences in humans in order to assess the implications for clinical practice.

The targeted intervention was a minimum dose of $25 \mathrm{mg}$ of trazodone daily, for at least 1 week, to observe the chronic effects of this drug and not the effects obtained after a single dose. Consequently, we excluded studies that only tested the acute effects of trazodone by using it in a one-time-only fashion. The dose of $25 \mathrm{mg}$ was chosen because it is the minimum dose used that is capable of producing some effects of the drug observed in clinical practice. In terms of comparators, all comparators were accepted without restriction.

Our main outcome was to study the effect of trazodone on human cognition. To this end, we analysed all instruments that measured the cognitive impairment that appeared in the studies included in the qualitative synthesis. These instruments included the Montreal Cognitive Assessment scale (MoCA), Mini-Mental State Examination (MMSE), the Digit Span subtest, the $\mathrm{d} 2$ test, the Wisconsin Card Sorting Test, Continuous Performance Test, n-back Test, Paired Associate Learning Test-Form I (short-term memory), Paired Associate Learning Test-Form II (long-term memory) of the Wechsler Memory Scale, Arithmetic, Letter-Number Sequencing, Digit Symbol-Coding, Symbol Search of the Wechsler Adult Intelligence scale (third edition, WAIS-III), Buschke Selective Reminding Test, the Brown-Peterson Memory Test, the Word Learning Test, the Memory Scanning Test, the Critical Flicker/Fusion (CFF) frequency, the Critical Tracking Task, the Divided Attention Test, the Visual Vigilance Test, Rey's Verbal Memory (RVM) test, the Guild Memory Test, Trail Making Test, free recall test, Corsi block test, category generation, "News" recall, Who's who? or matching to sample. Our secondary outcome was whether trazodone could be included in the treatment of neurodegenerative diseases in humans. To assess effects on neurodegeneration, we searched for reports of beneficial effects of trazodone in cognitive decline and studies including analyses of mechanisms through which that benefit could occur.

The studies included randomized controlled trials, nonrandomized trials, and retrospective and prospective cohort studies. No limits in language or publication year were applied.

The literature search was done in electronic databases from September 14 to September 22, 2020. The search was conducted in MEDLINE (1990-present), Web of Science (1999-present), and CENTRAL from Cochrane (1994-present). The last search was run on November 2, 2020.

The following search terms were used to search in PubMed: trazodone; memory; memories; cognition; cognitions; cognitive; cognitively. The detailed search strategy can be assessed in supplementary Table 1.

Studies were selected in two phases independently by two reviewers. In the first phase, articles were chosen by their title and abstract. In the second phase, the articles selected in the previous phase were read in full to determine eligibility for inclusion. Data were collected manually and independently by the two reviewers and synthesized in tables. Disagreements between reviewers were resolved by discussion and consensus. For each study included, the following information was collected: the study author(s), title, year of publication and design, the follow-up period, the study size, the population being studied, its age and sex, the intervention under study, the comparators used, and the outcome measures (Tables 1 and 2). The effects of trazodone on human cognition were synthesized using a table to represent all scores of the cognitive evaluation scales obtained in order to determine the final result of the effect. The final result was divided into three categories: no effect (defined as neither improving nor impairing cognition), positive effect (defined as cognition improvement or delayed cognitive decline), and negative effect (defined as cognition impairment).

To ascertain the risk of bias, the two reviewers worked blindly and independently. For a formal risk-of-bias assessment, the Cochrane risk-of-bias tool was used for randomized controlled trials and the ROBINS criteria for observational studies.

A level of 0.05 was considered significant.

This review is registered in the PROSPERO International prospective register of systematic reviews (registration number: CRD42020172577) and can be accessed at https://www. crd.york.ac.uk/PROSPERO/display_record.php?RecordID= 172577. However, due to the COVID-19 pandemic, the review protocol was published exactly as submitted.

\section{Results}

\section{Study characteristics}

A total of 16 studies were included in the final qualitative analysis of the review, four of which were observational studies, and 12 were experimental studies. From the search of the electronic databases, a total of 267 citations were found. However, after removing the duplicates, 218 studies remained and were screened. Of these, based on out-of-context titles and abstracts, 186 were excluded and 32 full-text articles were assessed for eligibility. 


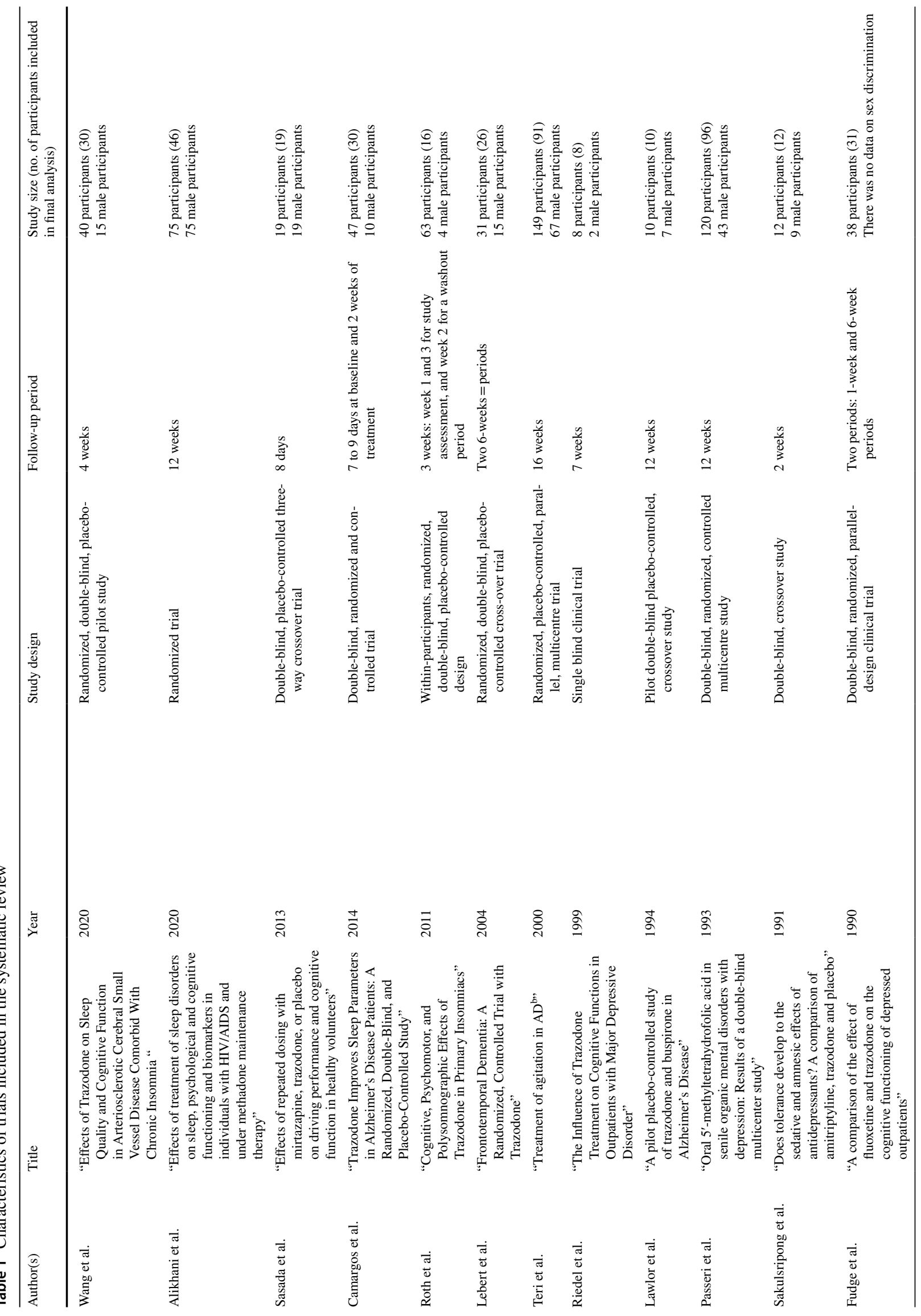




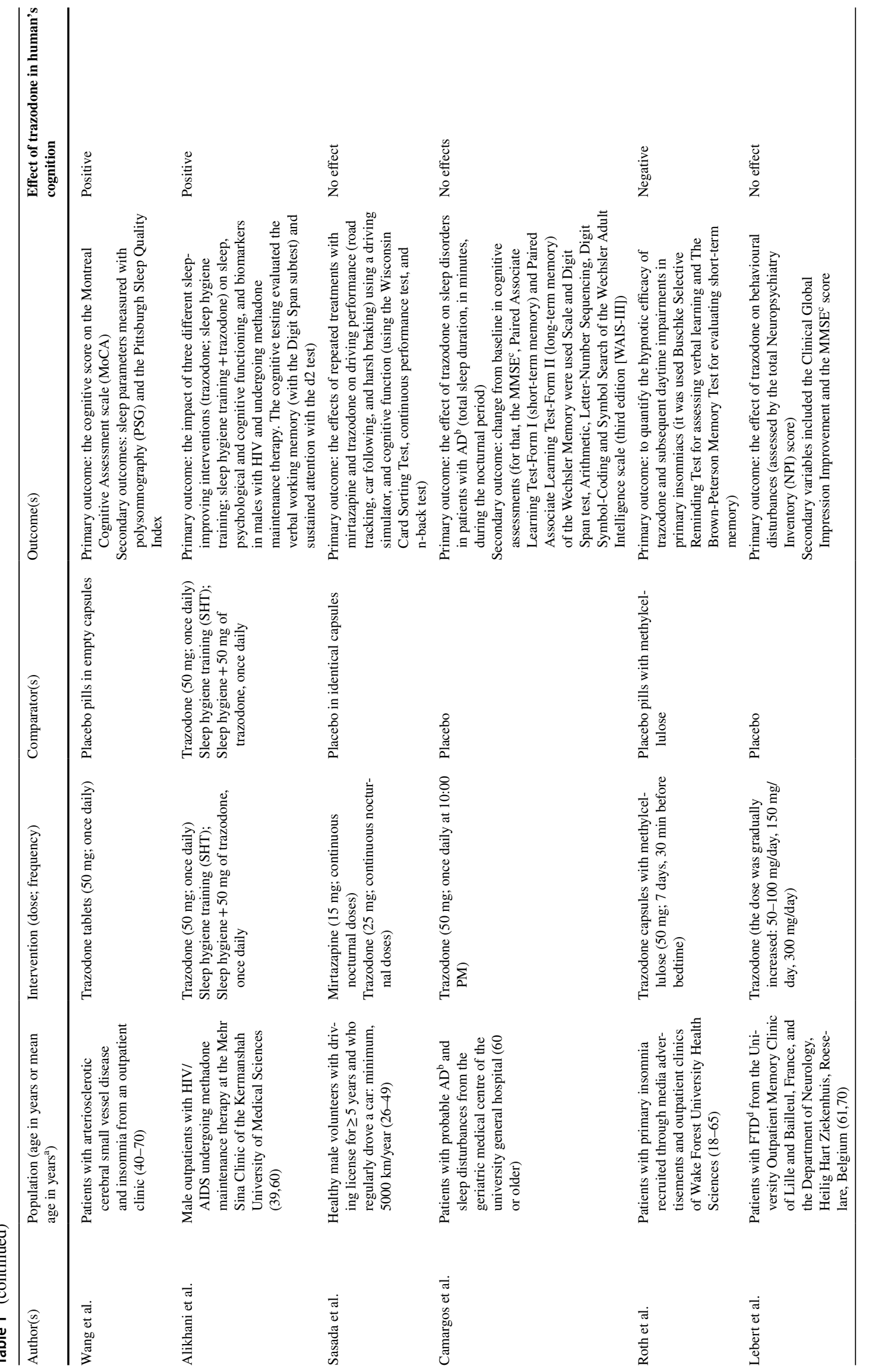




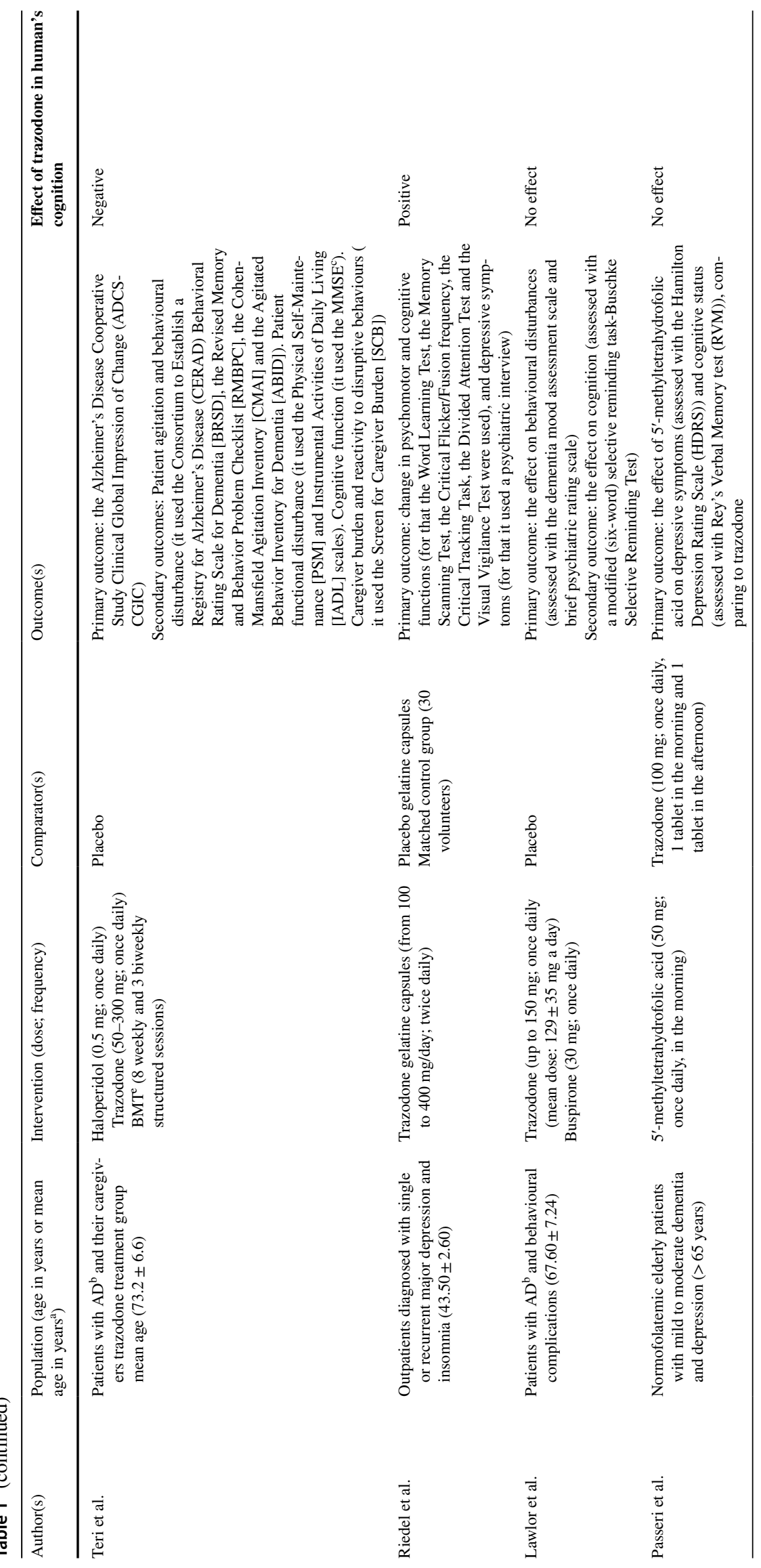




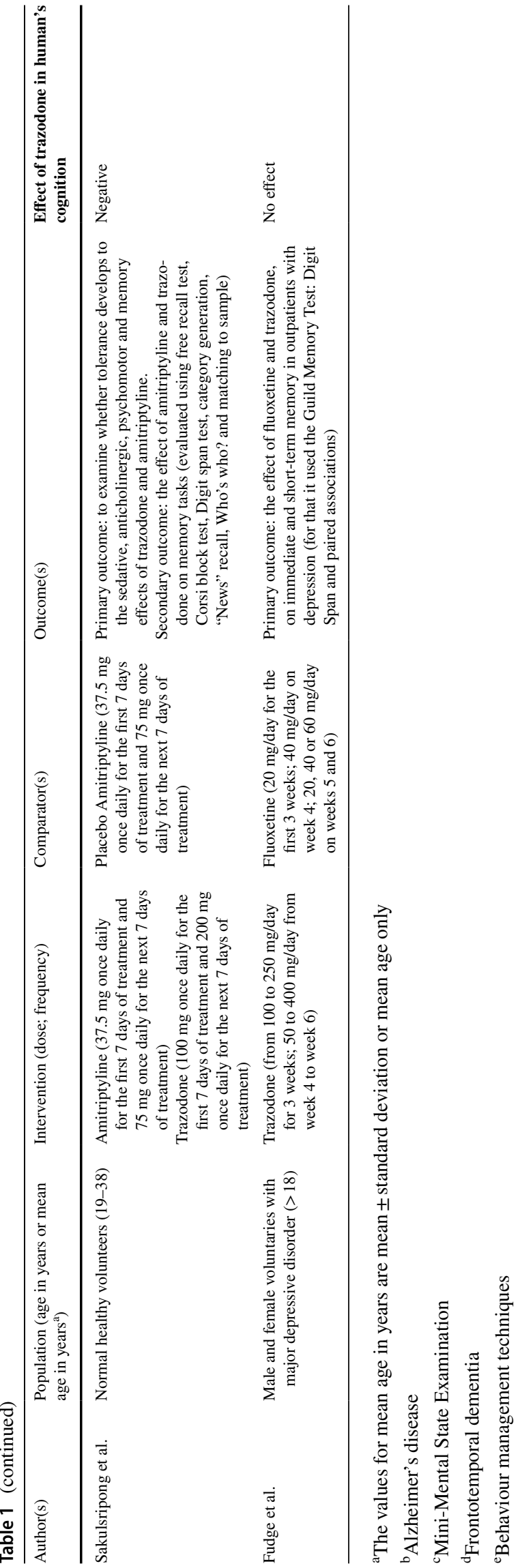

Sixteen articles were subsequently excluded for the following reasons: there were no data on outcomes of interest $(n=2)$, there was no follow-up period between trazodone use and effect assessment $(n=10)$, the study comprised only a commentary on an article $(n=1)$, an abstract $(n=1)$, or a letter to the editor $(n=1)$, or it was a randomized clinical trial in the recruiting phase $(n=1)$. Sixteen studies met the inclusion criteria and were included in the final analysis (see Supplementary Fig. 2).

For each study, the information collected is presented in Tables 1 and 2. The minimum and maximum follow-up periods observed were 8 days [7] and 16 weeks [8], respectively, for experimental studies, and 12 months [9] and 13 years [10], respectively, for observational studies (Tables 1 and 2).

Overall, the 16 studies included 8646 participants in the final analysis, with sample sizes ranging from 8 [11] to 6798 participants [10]. All studies assessed adult individuals at least 18 years of age. The majority included both men and women, with two studies (Sasada et al. and Alikhani et al.) including only men [7, 12], one (Leng et al.) including only women [13], and one (Fudge et al.) not differentiating by sex [14]. The participants were either healthy individuals or patients with one or more of the following conditions: arteriosclerotic cerebral small vessel disease (ASVD), insomnia, HIV/AIDS, dementia, Alzheimer's disease, frontotemporal dementia, and depression (Tables 1 and 2). Since studies included a wide age range, a higher prevalence of cognitive decline in older individuals should be expected, since cognition decreases with age.

The study designs included randomized trials $(n=10)$, non-randomized trials $(n=1)$, trials without reference to randomization $(n=1)$, retrospective cohorts $(n=2)$, and prospective cohorts $(n=2)$ published from 1990 to November 2020. Only three randomized controlled trials did not use a placebo in the comparator arm [12, 14, 15]. Intervention groups consisted of trazodone (with doses ranging from 25 to $400 \mathrm{mg}$ for a minimum of 1 week) alone or in association with sleep hygiene training (SHT) and mirtazapine $(15 \mathrm{mg})$. The other intervention groups included haloperidol (0.5 mg), behaviour management techniques (BMT), buspirone (30 mg), 5'-methyltetrahydrofolic acid (50 mg), amitriptyline (37.5-75 mg), sleep medications, zolpidem, benzodiazepines, tricyclic antidepressants, or noradrenaline reuptake inhibitors. Only one cohort study reported the doses used in the intervention groups [16] (Table 2).

All studies evaluated the effects of continuous doses of trazodone on cognition as a primary or secondary outcome. All other outcomes can be found in Tables 1 and 2.

\section{Effects on cognitive function}

The rightmost columns of Tables 1 and 2 display the ultimate effect of trazodone on human cognition. Overall, from the 16 studies included, seven showed no effect of 


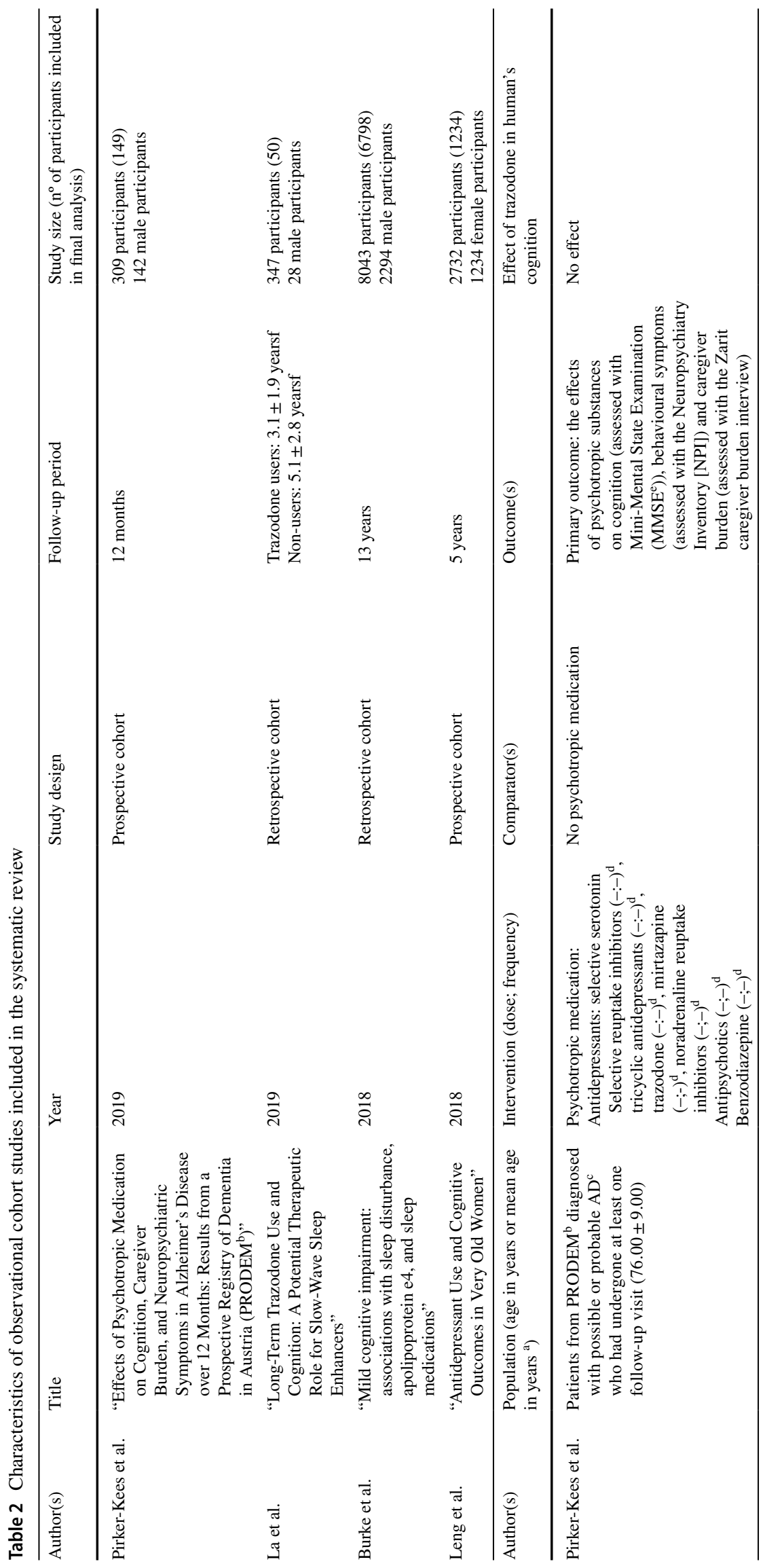




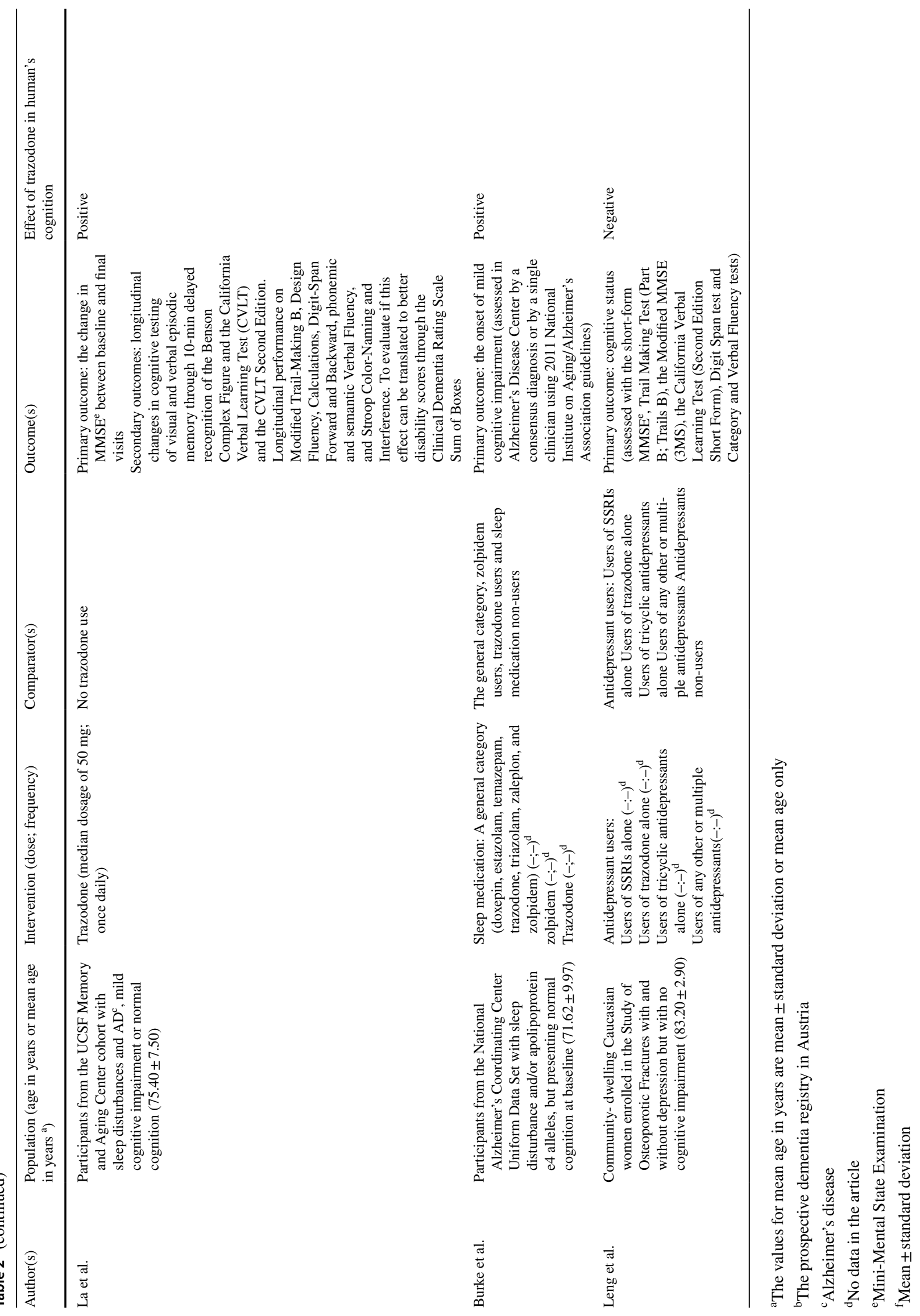


trazodone on human cognition [7, 9, 14, 15, 17-19], while five showed a positive effect by improving cognition or reducing cognitive decline $[6,10-12,16]$, and four showed a negative effect by impairing cognitive function $[8,13$, 20, 21].

Among studies with beneficial effects, Wang et al. demonstrated that trazodone significantly improved concentration and recall abilities in patients with ASVD and insomnia, with increased concentration and recall scores on MoCA after trazodone treatment compared to those at baseline [pre/post-treatment mean (SD) score in concentration and recall: $4.41(1.57) / 5.33(1.45)$ and 1.52 (1.03)/2.24 (1.12), respectively]. Alikhani et al. also showed that sustained attention on the $\mathrm{d} 2$ test was significantly improved from baseline to week 6 of treatment with trazodone alone or in combination with SHT [12]. Riedel et al. demonstrated that besides improving depressive symptoms during 5 weeks of treatment, trazodone lowered CNS arousal [CFF frequency was lower than during placebo periods: $F(1,7) 826, p<0.025]$ and improved delayed recall $[F(1,7) 25 \cdot 54, p<0.001$ in Word Learning Test], while no change was seen in tests that evaluated memory scanning, vigilance, tracking, and divided attention [11]. Furthermore, La et al. demonstrated an association between trazodone and delayed cognitive decline [16], and similar results were found by Burke et al. [10]. La et al. demonstrated that over an average follow-up period of 4 years, compared to trazodone users, trazodone non-users showed 2.6-fold faster decline in the MMSE score [16]. Burke et al. also showed that trazodone could potentially mitigate the risk of developing mild cognitive impairment (MCI) over a follow-up period of 13 years, decreasing cognitive decline in patients with sleep disorders and normal cognition at baseline.

In contrast, Roth et al. documented modest cognitive and motor impairments associated with trazodone, including a decline in short-term memory [Brown-Peterson Memory Test: main effect, $F(1,15)=17.3$, post hoc $t=4.2, p<0.001]$ and verbal learning [Selective Reminding Test: main effect, $F(1,15)=5.01, p=0.037$; post hoc $t=2.2, p=0.041]$ with time [20]. In concordance with these findings, Teri et al.. found a significant worsening in MMSE scores in the trazodone treatment group (change score means in MMSE $\pm \mathrm{SD}$ $:-1.97 \pm 3.15$ ) compared with BMT (change score means in $\mathrm{MMSE} \pm \mathrm{SD}-0.05 \pm 2.58$ ) after 16 weeks [8]. Sakulsripong et al. demonstrated that both amitriptyline and, albeit to a lesser extent, trazodone caused impairments in memory tasks such as free recall [recall trial $1 ; F(2,20)=15.6 ; p<0.001$, and recall trial $2 F(2,20)=8.6 ; p<0.012]$, short-term visual/ spatial memory [Corsi block test: $F(2,19)=6.79, p<0.01$ ], short-term verbal memory [Digit Span test: $F(2.19)=6.89$, $p<0.01$ ], and visual matching-to-sample only at $2 \mathrm{~h}$ of testing $[\mathrm{F}(1,20)=10.5, p<0.005]$, since these effects decreased over the 2-week period $[F(2,19)=4.5, p<0.05]$. Amitriptyline, but not trazodone, caused impairments in both immediate and delayed recall in "News" recall after a single dose [21]. In that respect, Leng et al. demonstrated that very elderly women using trazodone over a period of 5 years were three times as likely to progress to $\mathrm{MCI}$ and dementia when compared to non-trazodone users $(\mathrm{OR}=3.48 ; 95 \% \mathrm{CI}=1.12-10.81)$, even when excluding female participants with depressive symptoms $(\mathrm{OR}=6.11 ; 95 \% \mathrm{CI}=1.16-32.21)$ and after adjusting for baseline cognitive and depressive symptoms [13].

Among studies showing a neutral effect on cognition, Sasada et al. showed no effect of acute or repeated doses of trazodone on driving performance or cognitive function [7]. Camargos et al. demonstrated a therapeutic effect of trazodone on sleep disturbances in patients with Alzheimer's disease during a 2-week period, with no effects on cognition or functionality (namely, in activities of daily living) in these patients [17]. Lebert et al. demonstrated that over a 6-week period, and compared to placebo, trazodone showed no difference in the variation in MMSE scores $(p=0.1)$ [19]. Lawlor et al. demonstrated that, compared to placebo, treatments with both buspirone and trazodone for 4 weeks showed no significant change in free recall or new learning measures on the Buschke Selective Reminding Task (no numerical values were presented) [18]. Further, Passeri et al. showed that although the score on immediate recall assessed in RVM increased from $20 \pm 7$ to $23 \pm 8(p<0.01)$ after 8 weeks of treatment with 5 '-methyltetrahydrofolic acid, it remained unchanged within the trazodone group (from $22 \pm 9$ to $22 \pm 9$ ) [15]. For delayed recall, the score in the RVM test remained unchanged for both groups during the treatment period [15]. After a 4-week drug-free period, both treatments showed no changes in either immediate or delayed recall [15]. Fudge et al. evaluated the effects of fluoxetine and trazodone on the cognitive functioning of outpatients with depression, and found that neither drug affected cognitive skills on the Guild Memory Test over a period of 6 weeks [mean trazodone pair association (pair 1; pair 2) and Digit Span scores at baseline and after 6 weeks of treatment, respectively: baseline: $4.7+2.8$; $4.3+3.0$, and $12.2+2.4 ;$ after 6 weeks: $6.7+2.9 ; 6.3+3.3$, and $13.0+2.5$ ] [14]. Lastly, Pirker-Kees et al. showed that although MMSE declined over a 12-month follow-up period (MMSE: $21.2 \pm 4$ vs. $19.7 \pm 5, p=0.001)$, the individual treatment groups (including trazodone) did not change significantly over the same period ( $20.0 \pm 5$ vs. $19.9 \pm 5)$ [9].

\section{Other effects}

Most studies demonstrated the effects of trazodone in domains other than cognitive function. Wang et al., Alikhani et al., Camargos et al., and Roth et al. all showed that trazodone improved sleep quality and sleep parameters including 
sleep efficiency, N3 sleep ratio, and sleep continuity [6], daytime functioning [12], and nighttime sleep percent [17], while not inducing $[12,17]$ or decreasing daytime sleepiness $[6,20]$, reducing nighttime awakening [20], minutes of stage 1 sleep, or self-reports of trouble sleeping [20].

Lebert et al., Lawlor et al., and Pirker-Kees et al. also reported the beneficial role of trazodone in the treatment of behavioural disturbances in neurodegenerative diseases [9, $18,19]$. Teri et al. showed a modest reduction in agitation in patients with $\mathrm{AD}$ [8], while five studies revealed a decline and relief in symptoms of depression [11, 12, 14, 15] or anxiety [6, 12].

Roth et al. demonstrated a small impairment in equilibrium and arm muscle endurance [20], and Sasada et al. demonstrated that trazodone did not affect driving performance in healthy volunteers [7].

\section{Adverse events}

Only five studies mentioned the adverse effects of drugs used $[6,8,15,17,19]$. Overall, all study participants tolerated trazodone well, and adverse events were mild and not a major cause of participant drop-out. Similarly to previous studies, Wang et al. reported deterioration in insomnia, akathisia, nausea, loss of appetite, dizziness, and headache, all of mild intensity and none statistically different between trazodone and placebo treatment groups [6]. Camargos et al. did not specify the adverse events that occurred, but found no difference in frequency or severity rating of adverse events between trazodone and placebo groups [17]. Lebert et al. rated all adverse events as mild, leading to drop-out in only one patient [19]. In the placebo period, fatigue and dizziness were reported in three patients, while in the trazodone period, fatigue, dizziness, hypotension, and cold extremities were reported in 11 patients [19]. Passeri et al. reported blurred vision and vertigo in only one patient in the trazodone treatment group [15]. Lastly, Teri et al. reported agitation in the trazodone group (50\%), unacceptable adverse effects not otherwise specified in the haloperidol group (43\%), and increased agitation in the BMT group (35\%) as the main reasons for patient drop-out [8].

\section{Risk of bias}

Table 3 displays the results from the quality assessment of experimental studies using the Cochrane risk-of-bias tool. Of the 12 studies included, six had a low overall risk of bias, five had high risk, and one had some concerns. For the high-risk studies, the main problems arose from the randomization process, deviations from the intended interventions, and the selection of the reported results. A detailed evaluation is presented in Table 3 . For observational studies, Table 4 shows the results obtained using ROBINS-I criteria. Three studies presented an overall serious risk of bias mainly due to residual confounding and poor characterization of the intervention, and in one study it was not possible to reach a conclusion about the risk of bias due to lack of information about the concomitance of the beginning of the intervention and the follow-up (Table 4).

Table 3 Risk of bias of individual studies utilizing the Cochrane risk-of-bias tool

\begin{tabular}{|c|c|c|c|c|c|c|}
\hline \multirow[t]{2}{*}{ Studies } & \multicolumn{6}{|l|}{ Risk of bias } \\
\hline & $\begin{array}{l}\text { Randomization } \\
\text { process }\end{array}$ & $\begin{array}{l}\text { Deviations from the } \\
\text { intended interven- } \\
\text { tions }\end{array}$ & Missing outcome data & $\begin{array}{l}\text { Measurement } \\
\text { of the outcome }\end{array}$ & $\begin{array}{l}\text { Selection of } \\
\text { the reported } \\
\text { result }\end{array}$ & Overall risk of bias \\
\hline Wang et al. & Some concerns & Low risk & Low risk & Some concerns & Some concerns & Some concerns \\
\hline Alikhani et al. & Some concerns & Some concerns & Some concerns & Some concerns & Low risk & High risk \\
\hline Sasada et al. & Low risk & Low risk & Low risk & Low risk & Low risk & Low risk \\
\hline Camargos et al. & Low risk & Low risk & Low risk & Low risk & Low risk & Low risk \\
\hline Roth et al. & Some concerns & High risk & Low risk & Low risk & Low risk & High risk \\
\hline Lebert et al. & Low risk & Low risk & Low risk & Low risk & Low risk & Low risk \\
\hline Teri et al. & Low risk & Low risk & Low risk & Low risk & High risk & High risk \\
\hline Riedel et al. & High risk & High risk & Low risk & Low risk & Some concerns & High risk \\
\hline Lawlor et al. & Low risk & High risk & Low risk & Low risk & Low risk & High risk \\
\hline Passeri et al. & Low risk & Low risk & Low risk & Low risk & Low risk & Low risk \\
\hline $\begin{array}{l}\text { Sakulsripong } \\
\text { et al. }\end{array}$ & Low risk & Low risk & Low risk & Low risk & Low risk & Low risk \\
\hline Fudge et al. & Low risk & Low risk & Low risk & Low risk & Low risk & Low risk \\
\hline
\end{tabular}


Table 4 Risk of bias of individual studies utilizing ROBINS-I criteria for cohort studies

\begin{tabular}{|c|c|c|c|c|c|c|c|c|}
\hline \multirow[t]{2}{*}{ Studies } & \multicolumn{8}{|l|}{ Risk of bias } \\
\hline & Confounding & $\begin{array}{l}\text { Selection of } \\
\text { participants }\end{array}$ & $\begin{array}{l}\text { Classification } \\
\text { of interven- } \\
\text { tions }\end{array}$ & $\begin{array}{l}\text { Deviations } \\
\text { from the } \\
\text { intended } \\
\text { interventions }\end{array}$ & Missing data & $\begin{array}{l}\text { Measure- } \\
\text { ment of the } \\
\text { outcome }\end{array}$ & $\begin{array}{l}\text { Selection of } \\
\text { the reported } \\
\text { result }\end{array}$ & $\begin{array}{l}\text { Overall risk of } \\
\text { bias }\end{array}$ \\
\hline $\begin{array}{l}\text { Pirker-Kees } \\
\text { et al. }\end{array}$ & Moderate risk & Low risk & Serious risk & Low risk & Low risk & Low risk & Low risk & Serious risk \\
\hline La et al. & Moderate risk & $\begin{array}{l}\text { No informa- } \\
\text { tion }\end{array}$ & Low risk & Low risk & Low risk & Low risk & Low risk & No information \\
\hline Burke et al. & Moderate risk & $\begin{array}{l}\text { No informa- } \\
\text { tion }\end{array}$ & Serious risk & Low risk & Low risk & Low risk & Low risk & Serious risk \\
\hline Leng et al. & Moderate risk & Moderate risk & Serious risk & Low risk & Low risk & Low risk & Low risk & Serious risk \\
\hline
\end{tabular}

\section{Discussion}

To date, studies evaluating the effects of trazodone on human cognition have demonstrated diverse results, with some presenting a neutral or positive effect, and others displaying impaired cognition, making it difficult to reach a definitive conclusion. Overall, the majority of studies $(n=12)$ in our systematic review reported no effect or a positive effect of trazodone on cognitive function, while four demonstrated a negative result (Tables 1 and 2). Although our main findings suggest that trazodone does not impair human cognition, and may even have a beneficial effect, further studies are needed to confirm the overall effect of trazodone on cognitive function.

Wang et al., Alikhani et al., Riedel et al., La et al., and Burke et al. all demonstrated a beneficial effect of trazodone on cognition $[6,10-12,16]$. However, this positive result could be attributed to the positive effects of this drug on improvement in sleep disturbances such as insomnia $[6,10$, $12,16]$ and in depressive symptoms [11]. It is known that insomnia contributes to the progression of neurodegenerative diseases since it is associated with cognitive deterioration $[6,10,12]$. For that reason, it seems plausible that a drug, like trazodone, that increases sleep continuity and slow-wave sleep (SWS) ratio could consequently improve cognitive function [6]. Depressive symptoms are also related to cognitive decline [21], as it is associated with poor sleep and anxiety [12] (Figs. 1 and 2).

On the other hand, it is also known that antidepressants with muscarinic receptor antagonism activity can induce cognitive dysfunction [11]. However, in the study conducted by Riedel et al., high doses of trazodone (100-400 mg/day) improved memory and cognitive function in outpatients with depression $[11,22]$. The absence of a pronounced antimuscarinic effect observed with trazodone, even at higher doses, may be explained by the fact that this drug has the lowest antimuscarinic effects among anticholinergic antidepressants [11, 22]. On the other hand, Leng et al. found evidence of an association between trazodone and increased risk of cognitive impairment in elderly women without cognitive disturbances after 5 years, even when adjusting for baseline cognition and depressive symptoms, and after excluding participants with high depressive symptoms [13], suggesting a negative effect on cognition that is independent of improvement in depression. However, it is important to note that in this study, of the total number of participants $(n=1234)$, only 15 used trazodone alone. Additionally, since the study only included older Caucasian women, these results cannot not be generalized to different age, sex, and race groups [13]. For that reason, future studies should evaluate whether this association between trazodone use and cognitive decline is directly due to a negative effect of this antidepressant, or to the detrimental effects that depression exerts on cognitive function.

In summary, all studies that obtained a positive effect of trazodone on cognitive functions evaluated participants with ASVD, sleep disorders, HIV/AIDS while undergoing methadone maintenance therapy, major depression, $\mathrm{AD}$, or mild cognitive impairment-disorders frequently comorbid with insomnia and depression $[6,12]$. For that reason, the majority of these studies demonstrated a positive effect that is possibly not due to the direct effects of trazodone on cognition, but is instead mediated through an improvement in sleep disorders and depressive symptoms. Despite this, none of these results showed a negative effect on cognitive deterioration.

At the other end of the other spectrum, Roth et al. demonstrated a mild decremental effect on short-term memory and verbal learning with short-term low doses of trazodone $(50 \mathrm{mg})$ in patients with primary insomnia [20]. However, in concordance with studies mentioned above, trazodone improved cognitive function, providing an indirect beneficial effect in the treatment of insomnia. Nevertheless, the results obtained in Roth et al. are still plausible because, 


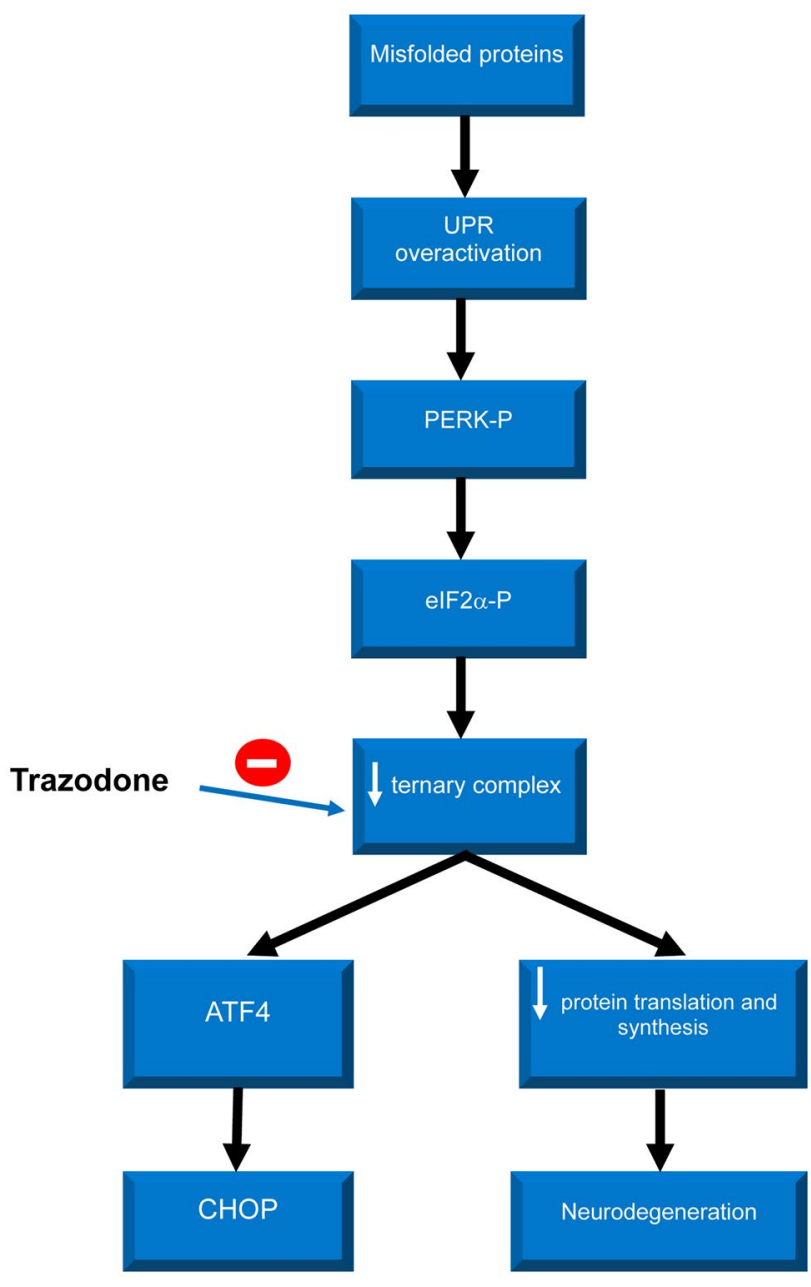

Fig. 1 UPR overactivation and trazodone site of action in the PERK branch of the UPR pathway. In protein misfolding disorders there is a disruption in protein homeostasis through endoplasmic reticulum (ER) stress, leading to the activation and dysregulation of the UPR response [5]. UPR acts as a cellular mechanism for the regulation of protein homeostasis when there are misfolded proteins [5] and coordinates this process through three ER transmembrane proteins: PERK, inositol-requiring enzyme 1 (IRE1), and activating transcription factor 6 (ATF6). Thus, in the face of misfolded proteins, PERK dimerizes, autophosphorylates, and becomes activated. Subsequently, PERK phosphorylates the $\alpha$-subunit of eIF2, preventing the formation of the ternary complex, resulting in a consequent blockage of proteins crucial for learning, memory, synaptic maintenance, and neuronal survival [5]. On the other hand, PERK activation culminates with the translation of activating transcription factor 4 (ATF4), which upregulates proteins that restore cellular homeostasis and CHOP [5]. Trazodone acts in the PERK branch of the UPR pathway downstream of eIF $2 \alpha-P$, preventing it from reducing levels of the ternary complex, allowing protein translation to occur [2], restoring neuronal protein synthesis rates, enabling a boost of memory and preventing neurodegeneration in mice models [5]. UPR: unfolded protein response; PERK-P: phosphorylated RNA (PKR)-like ER kinase; eIF2 $\alpha$ : $\alpha$-subunit of eukaryotic initiation factor 2; ATF4: activating transcription factor 4; CHOP: CEBP homologous protein as Wang et al. stated, acute sedation effects observed with trazodone treatment could impair cognitive function in the initial phases, and these effects could decrease with continuous therapy [6]. Roth et al.'s study only evaluated the acute effects of trazodone treatment (7 days), making it impossible to evaluate a potential improvement in cognitive test performance. For that reason, and as the authors noted, longterm treatment with this drug should be considered in future studies to better understand its true effects on human cognition. Along those lines, Teri et al. conducted a study with a follow-up period of 16 weeks [8] and found no difference in efficacy in the treatment of behavioural disturbances among trazodone, the other treatments (haloperidol and BMT), and placebo, demonstrating an additional decline in MMSE score with trazodone when compared to BMT [8]. Even so, other studies have demonstrated that this drug can improve behavioural symptoms $[18,19]$, making it impossible to exclude a positive effect of trazodone on cognitive function through a decrease in dementia behavioural disturbances. It is also important to note that despite failure to demonstrate a positive effect, neither of these studies showed either a sustained [20] or a direct negative effect $[8,20]$ of trazodone on human cognition. Finally, the negative memory effects observed in Sakulsripong et al. could be due to the timing of trazodone utilization: when given in the morning, trazodone impaired performance on memory tests due to sedation and impaired daily functioning [21].

In between these results, Sasada et al. demonstrated that $25 \mathrm{mg}$ of trazodone did not affect cognitive function [7]. One could argue that this low dose could be insufficient to have any repercussions on cognition; however, other studies included in this systematic review [14, 15, 17-19] also demonstrated no effect with higher doses, suggesting that there is no dose-dependent effect of trazodone on cognition. We also mentioned that acute sedation effects observed with trazodone treatment could impair cognitive function in initial phases [6]. However, in this study by Sasada et al., we also have a short follow-up period (8 days) with a relatively small population (19 participants), and the acute detrimental effects of trazodone are not shown. One explanation may be that the dose used in this study is just half of the dose used in the Roth et al. study $(50 \mathrm{mg})$. Another reason could be the younger, healthy population included in this study (healthy male volunteers aged 26-49 years) compared to the older and diseased participants included in Roth et al. (patients with primary insomnia, aged 18-65 years). These findings could suggest that acute impairments in cognition due to acute sedation effects observed with trazodone are related to diseased older individuals since "the elderly are more vulnerable to the side effects of pharmacological treatments" [7]. However, further studies are needed to better understand this acute effect. 
Fig. 2 PRISMA 2009 flow diagram

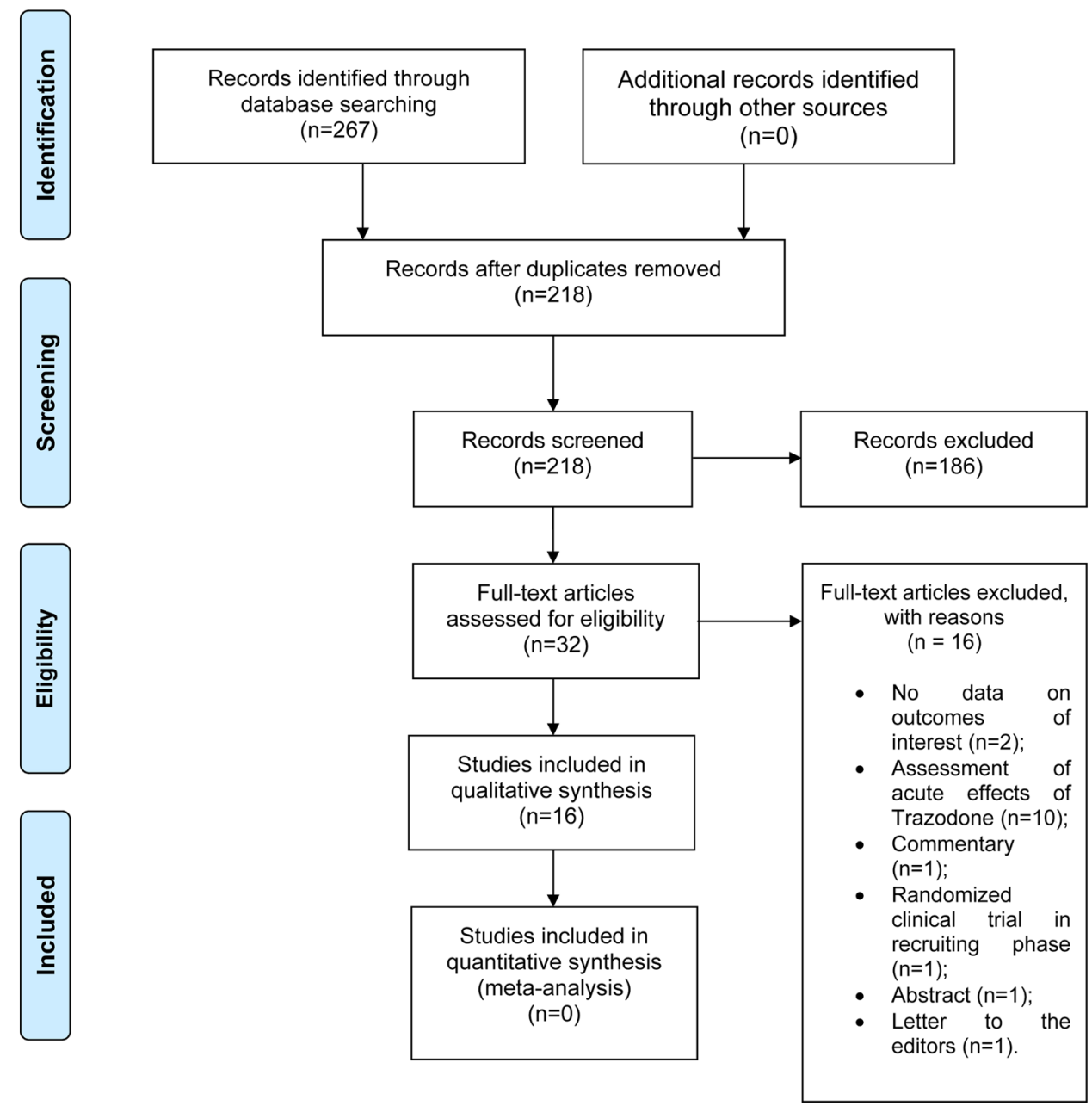

Lebert et al., Lawlor et al., Passeri et al., and Fudge et al. all demonstrate that even medium to high doses of trazodone (at least $100 \mathrm{mg}$ ) can be safely used in the treatment of behavioural disturbances in FTD [19] and AD [18], in depressive symptoms in mild to moderate dementia [15], and in outpatients with depression [14], as the studies showed no detrimental effects on cognition, along with good tolerability and minimal adverse events. Nevertheless, in contrast to Riedel et al., Passeri et al. did not show improved cognitive function through alleviation of depressive symptoms [15]. In comparison to Riedel et al., Passeri et al. had a long-term follow-up period (12 weeks), included more participants $(n=120)$ at an older age (more than 65 years), and used lower doses of trazodone $(100 \mathrm{mg})$. However, the authors stated that they did not completely understand why an improvement in depressive symptoms did not lead to a better score on cognitive function tests [15]. These results were corroborated by Fudge et al. [14]. Further studies with longer follow-up periods are needed to understand whether the absence of an effect on cognitive function demonstrated by these studies translates into the prevention of additional cognitive deterioration.
Another aspect worth mentioning is related to patients' caregivers. Stabilization of cognitive decline in patients with dementia is relevant not only to the patients themselves but also to their caregivers because, as reported by Pirker-Kees et al., caregiver burden increases with patients' cognitive decline [9]. Consequently, addressing cognitive decline also has a role in decreasing caregiver burden and preventing burnout.

A quite interesting result is shown in the study by La et al., which demonstrated an association between trazodone use and delayed cognitive decline in patients with normal cognition, $\mathrm{AD}$, and mild cognitive impairment, suggesting a potential role for its use in the treatment of dementia itself, and not only in dementia comorbidities such as insomnia and agitation [16]. However, the mechanism through which this was achieved was not explored [16]. In this study, trazodone use and the follow-up period were considerably longer than in other studies included in this review that showed no effect or negative effects of trazodone on cognition. These results demonstrate a longitudinal beneficial effect with long-term trazodone use that is not apparent after only a few weeks of utilization [16]. 
These results thus suggest that trazodone may possess a doubledose non-dependent effect on cognitive function: In acute use, it may impair cognitive function through its sedative acute effects. On the other hand, when used continuously for long periods, this drug may act on mechanisms that delay cognitive decline, effects that can be observed only over long periods of time [16]. These mechanisms could include improvement in SWS and prevention of UPR pathway overactivation as a result of both neurodegenerative diseases (Supplementary Fig. 1) and sleep deprivation [16]. The acute effects of trazodone may also be due to its actions on histamine $\mathrm{H} 1$ and muscarinic acetylcholine receptors. By contrast, its longitudinal effects on cognition could be ascribed to the rebalancing of serotonin levels, which is associated with a delayed onset of action.

It is also important to mention that we did not observe a dose-dependent effect of trazodone on cognition, since studies with low and high doses demonstrated either a positive $[6,10-12,16]$, negative $[8,13,20,21]$, or no effect $[7,9,14$, $15,17-19]$ of the drug on cognitive functions.

\section{Limitations}

This study has some limitations that warrant caution on the part of readers in interpreting our findings. First, all but four studies $[9,10,13,16]$ had short periods of trazodone utilization and follow-up of less than a year, preventing us from assessing whether the beneficial effects of trazodone could develop with continuous utilization, whether they were truly persistent over time, or whether the absence of effects could translate into prevention of additional cognitive deterioration. Second, the studies used a variety of tests to evaluate the effect of trazodone on cognition, making it difficult to compare results across studies. Third, most of the studies only evaluated the effect of trazodone in one or two cognitive domains, which makes it difficult to estimate an effect on human cognition as a whole. Fourth, three studies did not report the drug doses utilized [9, 10, 13]. Fifth, in one study, adverse effects were reported by participants spontaneously, some of whom had dementia, which could have led to misrecognition of side effects and reporting bias [17]. Also, in one experimental study, participants were aware of the intervention they received [12], leading to a possible influence on reporting the outcomes. Sixth, the majority of our experimental studies included few participants in each treatment arm. For that reason, future studies should incorporate a larger number of participants. Seventh, as a selective serotonin reuptake inhibitor, trazodone has a delayed onset of action. For that reason, the results of short-term studies are not adequately representative of its possible effects. Lastly, of all studies included in this systematic review that demonstrated a positive or detrimental effect on cognition, none explains the mechanisms through which this occurs, making it difficult to understand whether these effects are mediated through improvement in SWS, depression, and other behavioural disturbances, or whether they are attributable to a pharmacological action (such as acting in the UPR pathway) or to a combination of these effects, opening doors for future larger and long-term prospective studies to answer this question.

\section{Conclusion}

In conclusion, trazodone is a widely used old molecule with multiple mechanisms of action, some of which have been useful in treating depression, insomnia, agitation, and other behavioural disturbances [3], while others have been recently discovered, with benefits in the pathophysiological mechanism of neurodegeneration in mice [2]. Given its common use in the elderly population, where some degree of cognitive decline is expected, the establishment of its effects on cognition is of paramount importance to prevent accelerated cognitive decline, deterioration of patient quality of life, and caregiver burden. However, our results are not totally conclusive, since the cognitive consequences of trazodone are complex and require further studies to fully understand its overall effect on human cognition. Nevertheless, 12 of 16 clinical studies demonstrated a neutral or even a beneficial effect on cognition. This indicates that trazodone has no long-term detrimental effect on cognitive function, thus suggesting that despite being FDA-approved only for the treatment of depression [3], trazodone can be safely used in the treatment of comorbid conditions of patients with dementia, such as insomnia, agitation, and other behavioural symptoms.

Our results also highlight the possibility of a dose-independent dual effect of trazodone on human cognition, with acute utilization associated with impaired cognitive function and longitudinally long-term use with prevention of cognitive deterioration. None of the studies evaluated its effects on the UPR pathway, and there was no evidence that trazodone could be used as an active treatment of neurodegenerative diseases itself, although this review suggests that trazodone can be integrated into the therapeutic arsenal in these cases as a safe and well-tolerated adjuvant treatment for dementia comorbidities, with minimal adverse events.

For these reasons, and since only animal studies have been used so far to explore the effects of trazodone on the UPR pathway, future studies should be designed as large, prospective, double-blind randomized controlled trials that focus on evaluating the long-term repercussions of trazodone with regard to human cognition. Ideally, such studies should be done in subjects free of depression and insomnia, utilizing tests that represent all cognitive domains, and exploring the role of trazodone in the UPR pathway. We consider that further study of trazodone, an old molecule with new perspectives, is crucial for a better understanding of neurodegenerative mechanisms that could open doors for potential sites of action of future anti-dementia drugs. 
Supplementary information The online version contains supplementary material available at https://doi.org/10.1007/s00228-021-03161-6.

Authors' contributions Ana Mafalda Gonçalves Gonçalo contributed to the design and conceptualization of the study, and had a major role in the acquisition, analysis, and interpretation of the data. Maria Augusta Vieira-Coelho, MD, Ph.D. contributed to the design and conceptualization of the study, had a major role in the acquisition, analysis, and interpretation of the data, and revised the manuscript for intellectual content.

Funding The authors declare no funding for this study.

Availability of data and material All data collected and analyzed are available in this article.

\section{Declarations}

Conflict of interest The authors declare no conflict of interest.

\section{References}

1. Ashford JW (2019) Treatment of Alzheimer's Disease: Trazodone, Sleep, Serotonin, Norepinephrine, and Future Directions. J Alzheimer's Dis: JAD 67(3):923-930. https://doi.org/10.3233/ jad-181106

2. Halliday M, Radford H, Zents KAM, Molloy C, Moreno JA, Verity NC, Smith E, Ortori CA, Barrett DA, Bushell M, Mallucci GR (2017) Repurposed drugs targeting eIF2 $\alpha$-P-mediated translational repression prevent neurodegeneration in mice. Brain J Neurol 140(6):1768-1783. https://doi.org/10.1093/brain/awx074

3. Schwasinger-Schmidt TE, Macaluso M (2019) Other Antidepressants. Handb Exp Pharmacol 250:325-355. https://doi.org/10. 1007/164_2018_167

4. Ashford JW, Miller TW (1996) Effects of trazodone on sleep in patients diagnosed with post-traumatic stress disorder (PTSD). J Contemp Psychother 26(3):221-233. https://doi.org/10.1007/ BF02307588

5. Smith HL, Mallucci GR (2016) The unfolded protein response: mechanisms and therapy of neurodegeneration. Brain J Neurol 139(Pt 8):2113-2121. https://doi.org/10.1093/brain/aww101

6. Wang J, Liu S, Zhao C, Han H, Chen X, Tao J, Lu Z (2020) Effects of trazodone on sleep quality and cognitive function in Arteriosclerotic cerebral small vessel disease comorbid with chronic insomnia. Front Psych 11:620. https://doi.org/10.3389/ fpsyt.2020.00620

7. Sasada K, Iwamoto K, Kawano N, Kohmura K, Yamamoto M, Aleksic B, Ebe K, Noda Y, Ozaki N (2013) Effects of repeated dosing with mirtazapine, trazodone, or placebo on driving performance and cognitive function in healthy volunteers. Hum Psychopharmacol 28(3):281-286. https://doi.org/10.1002/hup.2321

8. Teri L, Logsdon RG, Peskind E, Raskind M, Weiner MF, Tractenberg RE, Foster NL, Schneider LS, Sano M, Whitehouse P, Tariot P, Mellow AM, Auchus AP, Grundman M, Thomas RG, Schafer K, Thal LJ (2000) Treatment of agitation in AD: a randomized, placebo-controlled clinical trial. Neurology 55(9):1271-1278. https://doi.org/10.1212/wnl.55.9.1271

9. Pirker-Kees A, Dal-Bianco P, Schmidt R (2019) Effects of psychotropic medication on cognition, caregiver burden, and neuropsychiatric symptoms in Alzheimer's disease over 12 months: results from a prospective registry of dementia in Austria (PRODEM). J Alzheimer's Dis: JAD 71(2):623-630. https://doi. org/10.3233/jad-181102

10. Burke SL, Hu T, Spadola CE, Li T, Naseh M, Burgess A, Cadet $\mathrm{T}$ (2018) Mild cognitive impairment: associations with sleep disturbance, apolipoprotein e4, and sleep medications. Sleep Med 52:168-176. https://doi.org/10.1016/j.sleep.2018.09.001

11. Riedel WJ, Schoenmakers E, Vermeeren A, O'Hanlon JF (1999) The influence of trazodone treatment on cognitive functions in outpatients with major depressive disorder. Hum Psychopharmacol Clin Exp 14 (7):499-508

12. Alikhani M, Ebrahimi A, Farnia V, Khazaie H, Radmehr F, Mohamadi E, Davarinejad O, Dürsteler K, Sadeghi Bahmani D, Brand S (2020) Effects of treatment of sleep disorders on sleep, psychological and cognitive functioning and biomarkers in individuals with HIV/AIDS and under methadone maintenance therapy. J Psychiatr Res 130:260-272. https://doi.org/10.1016/j.jpsychires. 2020.07.043

13. Leng Y, Diem SJ, Stone KL, Yaffe K (2018) Antidepressant use and cognitive outcomes in very old women. J Gerontol A Biol Sci Med Sci 73(10):1390-1395. https://doi.org/10.1093/gerona/ glx226

14. Fudge JL, Perry PJ, Garvey MJ, Kelly MW (1990) A comparison of the effect of fluoxetine and trazodone on the cognitive functioning of depressed outpatients. J Affect Disord 18(4):275-280. https://doi.org/10.1016/0165-0327(90)90079-n

15. Passeri M, Cucinotta D, Abate G, Senin U, Ventura A, Stramba Badiale M, Diana R, La Greca P, Le Grazie C (1993) Oral 5 '-methyltetrahydrofolic acid in senile organic mental disorders with depression: results of a double-blind multicenter study. Aging (Milan, Italy) 5(1):63-71. https://doi.org/10.1007/bf03324128

16. La AL, Walsh CM, Neylan TC, Vossel KA, Yaffe K, Krystal AD, Miller BL, Karageorgiou E (2019) Long-term trazodone use and cognition: a potential therapeutic role for slow-wave sleep enhancers. J Alzheimer's Dis: JAD 67(3):911-921. https://doi.org/10. 3233/jad-181145

17. Camargos EF, Louzada LL, Quintas JL, Naves JO, Louzada FM, Nobrega OT (2014) Trazodone improves sleep parameters in Alzheimer disease patients: a randomized, double-blind, and placebocontrolled study. Am J Geriatr Psychiatry 22(12):1565-1574. https:// doi.org/10.1016/j.jagp.2013.12.174

18. Lawlor BA, Radcliffe J, Molchan SE, Martinez RA, Hill JL, Sunderland T (1994) A pilot placebo-controlled study of trazodone and buspirone in alzheimer's disease. Int J Geriatr Psychiatry 9(1):55-59. https://doi.org/10.1002/gps.930090112

19. Lebert F, Stekke W, Hasenbroekx C, Pasquier F (2004) Frontotemporal dementia: a randomised, controlled trial with trazodone. Dement G

eriatr Cogn Disord 17(4):355-359. https://doi.org/10.1159/000077171

20. Roth AJ, McCall WV, Liguori A (2011) Cognitive, psychomotor and polysomnographic effects of trazodone in primary insomniacs. J Sleep Res 20(4):552-558. https://doi.org/10.1111/j.13652869.2011.00928.x

21. Sakulsripong M, Curran HV, Lader M (1991) Does tolerance develop to the sedative and amnesic effects of antidepressants? A comparison of amitriptyline, trazodone and placebo. Eur J Clin Pharmacol 40(1):43-48. https://doi.org/10.1007/bf00315137

22. El-Fakahany E, Richelson E (1983) Antagonism by antidepressants of muscarinic acetylcholine receptors of human brain. Br J Pharmacol 78(1):97-102 